\title{
Understanding Digital Information Needs of Supportive Care for Chinese Children with Cancer and Their Families: The Perspectives of Multi-Stakeholders
}

\author{
Lei CHENG $^{\mathrm{a}, 1}$, Zhang ZIHE ${ }^{\mathrm{a}, 2}$ and Shen XIN ${ }^{\mathrm{b}, 3}$ \\ ${ }^{a}$ School of Nursing, Fudan University, Shanghei, China \\ ${ }^{\mathrm{b}}$ School of Basic Medical Sciences, Fudan University, Shanghai, China
}

Keywords. Children, cancer, digital information needs, multi-stakeholders, supportive care

\section{Introduction}

Children with cancer and their families endure multiple challenges during treatment and also in survivorship. Digital health technologies provide an innovative way to support their needs. However, there remains gap about the digital information needs of supportive care for Chinese children with cancer and their families, especially from the perspectives of multi-stakeholders. This study aimed to understand the digital information needs by interviewing pediatric cancer patients, their families, nurses and physicians, which provide the foundation for developing a digital platform.

The project has been approved by the ethics committee of Shanghai Medical College of Fudan University（IRB\#2019-02-06Ethics Committee of School of Nursing, Fudan University, Shanghai,China).This study was supported by Fudan University-Shanghai Medical College-Qingfeng Project, China National Natural Science Foundation of China Youth Science Foundation (71904030), Shanghai Pujiang Talent Program (2019PJC006).

\section{Approach}

Semi-structured interviews were conducted with a purposive sample of multiple stakeholders (children, families, nurses and physicians) in the Hematology and Oncology department of Children' s Hospital of Fudan University, Shanghai, China in January, 2021. Questions in interviews guide included: understanding of their disease (for children and parents); the digital information they currently got (for children and parents)/being provided (for nurses and physicians) during previous treatment experience; perceptions and comments about the current digital information provided;

\footnotetext{
${ }^{1}$ Corresponding Author, Lei Cheng, PhD, RN, School of Nursing, Fudan University, Shanghai, China; E-mail: chenglei@fudan.edu.cn.
} 
digital information needs that were met during each treatment stage; suggestions for developing a digital platform to support Chinese children with cancer and their families. All the interviews were recorded and transcribed verbatim. We used a qualitative content analysis where themes and categories are obtained from the data on the context of the pre-determined questions. Transcripts were independently reviewed and coded by all three authors and an additional research assistant. After coding, we worked together to find consensus on the themes.

\section{Body}

A total of 6 patients, 12 parents, and 1 physician and 5 nurses were recruited. The subjects of the study included 6 children aged 7 to 10, mainly diagnosed with acute leukemia for an average of 1 year. Five nurses aged 27-38 with mainly worded for hematology oncology for an average of 7.6 years. A physician with doctor degree has worded for two years. Of the 13 parents, three of them are fathers, and the remaining ten are mothers, with different degree from junior high school to bachelor degree.

Three themes and ten subthemes emerged from the data. Theme 1 "the main contents of digital information needs": Subtheme 1 disease and treatment information; Subtheme 2 caring information; Subtheme 3 mental health information; Subtheme 4 finical support information. Theme 2 "the way of meeting digital information needs": Subtheme 5 accompanying cancer trajectory; Subtheme 6 in and outside of the hospital. Theme 3 "factors influencing digital information needs": Subtheme 7 disease factors; Subtheme 8 family factors; Subtheme 9 digital planforms; Subtheme 10 community factors.

\section{Conclusions}

This study shows that the information needs of children with hematological tumors and their parents in participating in family supportive care for children have not been fully satisfied. There is an urgent need for us to take the needs of children and their parents as a starting point and carry out a targeted digital platform to support them.

According to different stages of illness and different care situations inside and outside the hospital, comprehensively provide information including medication, treatment, in-hospital and home care of the child, and provide interesting and easy-tounderstand targeted information to the child and the parent of the child in different tones. We will conduct a more in-depth study on this in the next stage.

\section{Acknowledgement}

This study was supported by Fudan University-Shanghai Medical College-Qingfeng Project (), China National Natural Science Foundation of China Youth Science Foundation (71904030), Shanghai Pujiang Talent Program (2019PJC006).

The authors would like to thank all the participants. 


\section{References}

[1] Rodriguezgalindo C, Friedrich P, Alcasabas P et al. Toward the cure of all children with cancer through collaborative efforts: Pediatric oncology as a global challenge [J]. Journal of Clinical Oncology Official Journal of the American Society of Clinical Oncology, 2015, 33(27):3065-3073.

[2] Linder LA, Al-Qaaydeh S, Donaldson G. Symptom characteristics among hospitalized children and adolescents with cancer [J]. Cancer Nurs, 2018, 41(1): 23-32.

[3] Fitch MI. Providing supportive care for individuals living with cancer (Task Force Report). Toronto: Ontario Cancer Treatment and Research Foundation. 1994.

[4] Pelentsov LJ, Laws TA, Esterman AJ. The supportive care needs of parents caring for a child with a rare disease: A scoping review. Disabil Health J, 2015,8(4):475-491.

[5] U.S. Department of Health and Human Services, F.A.D.A. Guidance for industry: Patient-reported outcome measures: Use in medical product development to support labeling claims: draft guidance [J]. Health Qual Life Outcomes, 2006, 4:79.

[6] Howell D, Li M, Sutradhar R et al. Integration of patient-reported outcomes (PROs) for personalized symptom management in "real-world" oncology practices: A population-based cohort comparison study of impact on healthcare utilization [J]. Supportive Care in Cancer, 2020: 1-10.

[7] Snyder CF, Jensen RE, Segal JB et al. Patient-reported outcomes (PROs): Putting the patient perspective in patient-centered outcomes research [J]. Medical care, 2013, $51(803)$ : S73.

[8] Devine KA, Viola AS, Coups EJ, Wu YP. (2018) Digital health interventions for adolescent and young adult cancer survivors. JCO clinical cancer informatics 2:1-15. doi:10.1200/cci.17.00138

[9] Borrelli B, Ritterband LM. (2015) Special issue on eHealth and mHealth: Challenges and future directions for assessment, treatment, and dissemination. Health psychology: official journal of the Division of Health Psychology, American Psychological Association 34s:1205-1208. doi:10.1037/hea0000323

[10] McCann L, McMillan KA, Pugh G. (2019) Digital interventions to support adolescents and young adults with cancer: Systematic review. JMIR cancer 5 (2):e12071. doi:10.2196/12071

[11] Ramsey WA, Heidelberg RE, Gilbert AM, Heneghan MB, Badawy SM, Alberts NM. (2019) eHealth and mHealth interventions in pediatric cancer: A systematic review of interventions across the cancer continuum. Psycho-oncology. doi:10.1002/pon.5280

[12] Oh H, Rizo C, Enkin M, Jadad A. (2005) What is eHealth (3): A systematic review of published definitions. Journal of medical Internet research 7 (1):e1. doi:10.2196/jmir.7.1.e1

[13] Wesley KM, Fizur PJ. (2015) A review of mobile applications to help adolescent and young adult cancer patients. Adolescent health, medicine and therapeutics 6:141-148. doi:10.2147/ahmt.s69209

[14] Roberts AL, Fisher A, Smith L, Heinrich M, Potts HW. (2017) Digital health behaviour change interventions targeting physical activity and diet in cancer survivors: A systematic review and metaanalysis. Journal of cancer survivorship: research and practice 11 (6):704-719. doi:10.1007/s11764-0170632-1

[15] Boulos MN, Wheeler S, Tavares $\mathrm{C}$ et al. How smartphones are changing the face of mobile and participatory healthcare: An overview, with example from eCAALYX [J]. Biomedical Engineering nline, 2011,10 .

[16] Mehl G, Labrique A. Prioritizing integrated mHealth strategies for universal health coverage [J]. Science,2014,345(6202):1284-1287.

[17] Patrick K, Griswold WG, Raab F et al. Health and the mobile phone [J]. American Journal f Preventive Medicine,2008,35(2):177-181.

[18] Klasnja P, Pratt W. Healthcare in the pocket: Mapping the space of mobile-phone health interventions [J]. Journal of Biomedical Informations,2012,45(1):184-198.

[19] Kahn JG, Yang JS, Kahn JS. 'Mobile' health needs and opportunities in developing countries [J]. Health Affairs,2010,29(2):252-258. 